\title{
Mechanical behavior of yttria-stabilized tetragonal zirconia polycrystal: Effects of different aging regimens
}

\section{Luís Felipe GUILARDI(a) Gabriel Kalil Rocha PEREIRA(b) Vinícius Felipe WANDSCHER ${ }^{(c)}$ Marília Pivetta RIPPE(d) Luiz Felipe VALANDRO(d)}

(a) Universidade Federal de Santa Maria UFSM, Faculty of Odontology,

Santa Maria, RS, Brazil.

(b)Faculdade Medidional - IMED, School of Dentistry, Passo Fundo, RS, Brazil.

(c) Centro Universitário Franciscano - Unifra, Faculty of Odontology, Prosthodontic Unit, Santa Maria, RS, Brazil.

(d) Universidade Federal de Santa Maria - UFSM, Faculty of Odontology,

Prosthodontic Unit, Santa Maria, RS, Brazil.

Declaration of Interest: The authors certify that they have no commercial or associative interest that represents a conflict of interest in connection with the manuscript.

Corresponding author:

Luiz Felipe Valandro

E-mail: Ifvalandro@hotmail.com

https://doi.org/10.1590/1807-3107bor-2017.vol31.0094

Submitted: July 6, 2017

Accepted for publication: Sep 11, 2017

Last revision: Oct 02, 2017
Abstract: This study aimed to characterize and compare the effect of different aging regimens on surface characteristic (topography and roughness), structural stability (phase transformation) and mechanical performance (Weibull analysis) of a Y-TZP ceramic. Discs $(15 \times 1.2 \mathrm{~mm}$; VITA In-Ceram YZ) were prepared according to ISO 6872-2015 for biaxial flexural strength testing and randomly assigned into five groups $(n=30)$ : as-sintered, no aging treatment (CTRL); $20 \mathrm{~h}$ in autoclave at $134^{\circ} \mathrm{C}, 2 \mathrm{bar}$ pressure (AUT); intermittent mechanical loading at $20 \mathrm{~Hz} / 10^{6}$ load pulses (MechLoad); AUT followed by MechLoad (AUT+MechLoad); and storage in distilled water at $37^{\circ} \mathrm{C}$, for 1 year (STO). The following analyses were performed: roughness $(n=30)$, surface topography $(n=2)$, phase transformation $(n=2)$ and biaxial flexure strength $(n=30)$. Phase transformation (increase of m-phase content) was shown to be a spontaneous, unavoidable and time-dependent process, occurring even under ambient conditions (dry storage after 1 year $=6.0 \%$ increase), and is considerably accelerated in the presence of moisture $(\mathrm{STO}=17.6 \%$; $\mathrm{AUT}=63.1 \%$; and AUT+MechLoad $=59.9 \%$ ). For roughness parameters, only $R a$ was affected by aging, and the highest values were observed for AUT+MechLoad $(0.25 \pm 0.07 \mu \mathrm{m})$. For Weibull analysis, structural reliability (Weibull moduli) and characteristic strength were not impaired after aging, and some aging conditions led to increased values (highest weibull moduli in AUT, and highest characteristic strength in STO). Phase transformation proves to be a time-dependent spontaneous mechanism that is accelerated in the presence of different stimuli. However, none of the aging regimens had a negative effect on the characteristic strength and structural reliability of Y-TZP ceramic.

Keywords: Dental Ceramics. Zirconium Oxide Partially Stabilized by Yttrium. Low-Temperature Degradation. Mechanical Properties. Phase Transformation.

\section{Introduction}

Yttria-stabilized tetragonal zirconia polycrystal (Y-TZP) ceramic has been widely used in prosthetic dentistry as frameworks for single or multi-unit fixed dental prostheses (FDPs) and as monolithic fullcontour restorations, being an alternative to the traditional metal-ceramic restorations. ${ }^{1} \mathrm{Y}$-TZP is a metastable material with a high crystalline content, 
consisting mainly of $0.2-0.5 \mu \mathrm{m}$ diameter uniform equiaxed grains prepared from fine $\mathrm{ZrO}_{2}$ (zirconia) particles and 3.5-8.7 mol\% of $\mathrm{Y}_{2} \mathrm{O}_{3}$ (yttria) stabilizer, which stabilizes the zirconia in its tetragonal phase. ${ }^{2}$

The tetragonal phase $(\mathrm{t})$ stabilization at room temperature does not completely prevent its transformation into the monoclinic phase (m). Different stimuli (mechanical, physical and/or chemical) can trigger this transformation. ${ }^{3}$ The $\mathrm{t}-\mathrm{m}$ phase transformation results in a volumetric increase of about $4 \%$ in grain size that creates a local compressive stress capable of hindering crack propagation. This mechanism, known as "transformation toughening mechanism", increases the material toughness and fatigue threshold. ${ }^{4}$

Even though the $\mathrm{t}-\mathrm{m}$ phase transformation has a positive initial effect (toughening), ${ }^{4,5,6}$ it may spread into the material structure through a nucleation-and-growth mechanism, ${ }^{3}$ leading to grain detachment, increased roughness, and production of cracks and micro cracks, which jeopardize strength, fracture toughness and density of the Y-TZP. ${ }^{3}$ This mechanism is known as low-temperature degradation (LTD). ${ }^{7}$

Theoretically, LTD is a time-dependent phenomenon that occurs faster when the Y-TZP is submitted to a humid environment with constant temperature changes. ${ }^{2,6,8}$ In general, LTD has been studied under specific aging conditions (storage in distilled water, storage in acidic solutions, autoclave cycles, mechanical cycling, etc.). ${ }^{2,9,10,11}$ Only a few studies ${ }^{6,12,13,14}$ assessed combined aging treatments (autoclave + mechanical cycling; thermal + mechanical cycling) to simulate more accurately the aging process in a clinical condition. Moreover, to the best of the authors' knowledge, no study has evaluated phase transformation in response to the long-term storage in moisture and in ambient conditions.

Therefore, the purpose of the present in vitro study was: (1) to characterize the Y-TZP ceramic behavior under different aging regimens, including combinations of regimens; and (2) to follow-up the effect of long-term under water storage on phase transformation evolution. The study hypothesis was that aging processes would increase the $\mathrm{m}$-phase content leading to a deleterious effect on the surface characteristics, flexural strength and structural reliability of the material.

\section{Materials and methods}

\section{Specimen preparation}

Disc-shaped specimens of Y-TZP (VITA In-Ceram YZ for inLab 40/19, VITA, Bad-Säckingen, Germany) were manufactured, according to ISO $6872-2015^{15}$ for biaxial flexural strength, using the methodology previously described by Guilardi and collaborators. ${ }^{16}$

In summary, two metal cylinder guides with 18 $\mathrm{mm}$ diameter were attached parallel to both sides of a Y-TZP block and then the lateral surfaces were ground using a polishing machine (EcoMet/AutoMet 250, Buehler, Lake Bluff, USA) with 600 grit silicon carbide sand paper until a cylinder was shaped. Afterward, the cylinders were cut under water irrigation with a diamond saw (ISOMET 1000, Buehler) in slices of 1.65 mm thickness and polished with a 1200-grit silicon carbide sand paper (on both sides).

Finally, the specimens were sintered as recommended by the manufacturer $\left(1,530^{\circ} \mathrm{C}\right.$; holding time of $25 \mathrm{~min}$; slow cooling until $400^{\circ} \mathrm{C}$ ), and then inspected with a digital caliper (Mitutoyo ABSOLUTE 500-196-20 Digital Caliper; Takatsu-ku, Kawasaki, Kanagawa, Japan) to guarantee that they were within the range recommended by ISO $6872-2015^{15}$ (diameter of $12-15 \mathrm{~mm}$; thickness of $1.2 \pm 0.2 \mathrm{~mm}$ ). After, specimens were randomly assigned into five different groups ( $n=30$ per group) according to the different aging regimens.

\section{Aging regimens}

a. CTRL: control group - "as-sintered" - no aging was performed.

b. STO: stored in a sealed vessel submerged in distilled water at $37{ }^{\circ} \mathrm{C}$ for 1 year in a steam chamber (Laboratory Thermo incubator - FANEM, São Paulo, Brazil).

c. AUT: 20 hours in a steam autoclave (Sercon HS1$\left.0300 \mathrm{n}^{\circ} 1560389 / 1\right)$ at $134^{\circ} \mathrm{C}$ under 2 bar pressure.

d. MechLoad: mechanical cycling - axial load application of $50 \%$ (444.82 MPa) of the mean biaxial flexural strength from the CTRL group, frequency of $20 \mathrm{~Hz}$ for $10^{6}$ cycles in an electrodynamic fatigue simulator (Instron ElectroPuls E3000, Instron Corporation, USA). The required load (in Newtons) to achieve the desired stress level 
was calculated according to ISO $6872-2015^{15}$, considering the thickness of each specimen.

e. AUT+MechLoad: combination of AUT and MechLoad methods described above. The only difference was that the $50 \%(496.68 \mathrm{MPa})$ of the mean biaxial flexural strength used was obtained from the AUT group.

\section{Phase transformation analysis}

The phase analysis was conducted $(n=2)$ using an X-ray diffractometer ( $2 \theta$ range of 25 to 35 degrees; scan time of $1 \mathrm{sec} / \mathrm{step}$, at $40 \mathrm{kV}, 40 \mathrm{~mA}$, step size of $0.03^{\circ}$; Bruker AXS, D8 Advance, Karlsruhe, Germany), through the Garvie \& Nicholson method modified by Toraya and collaborators, ${ }^{17}$ to determine the amount of $m$-phase content observed after each aging condition. This method has been extensively used ${ }^{8}$ and described in the literature. ${ }^{6,16}$ To assess the effect of time and to follow the $\mathrm{t}-\mathrm{m}$ phase transformation mechanism during under water storage, additional specimens $(n=2)$, subjected to storage under ambient conditions (room temperature $\pm 25^{\circ} \mathrm{C}$, without moisture, in a sealed plastic vessel), were made for baseline comparisons.

\section{Roughness analysis}

A micrometric analysis, consisting of three measurements for each specimen, was performed in a surface roughness tester ( $n=30$, Mitutoyo SJ-410, Mitutoyo), considering the $R a$ and $R z$ parameters (cut-off of $5 ; \lambda C$ of $0.8 \mathrm{~mm} ; \lambda S$ of $2.5 \mu \mathrm{m}$ ).

\section{Surface topography}

A Field Emission Scanning Electron Microscopy analysis (FE-SEM Inspect F50, FEI, North America Nano Port, Hillsboro, Oregon, USA) was executed in one specimen per group, after gold-palladium alloy sputtering, and the images were obtained at $10,000 \times$ and $50,000 \times$ magnifications. To assess the effect of aging on Y-TZP grain size, a computational measuring software (Image Tool3.0) was used to analyze the FE-SEM images and to determine the maximum and minimum values of grains size for each evaluated regimen.

\section{Biaxial flexural strength test}

Biaxial flexural strength $(n=30)$ was measured using a piston-on-three-ball assembly according to
ISO 6872-2015. ${ }^{15}$ Each specimen was immersed in water and the load was applied using a universal mechanical testing machine (load-to-failure at $1 \mathrm{~mm}$ / min; EMIC DL 2000, São José dos Pinhais, Brazil) until catastrophic failure, as previously described in many studies. $5,6,16$

\section{Qualitative fractographic analysis}

Fractured specimens were first analyzed in a light microscope (Stereo Discovery V20, Carl-Zeiss; Gottingen, Germany), and representative fractures were chosen for evaluation in FE-SEM (FEI Inspect F50; FEI) under $150 \times$ and $1,000 \times$ magnification to determine fracture origin and direction of crack propagation.

\section{Data analyses}

Due to its non-parametric distribution (Shapiro-Wilk test for normality and Levene's test for homoscedasticity), the micrometric roughness data ( $R a$ and $R z$ ) were analyzed through Kruskal-Wallis and LSD post-hoc tests.

The Weibull analysis was used to describe the reliability of the ceramic material. ${ }^{18}$ Through this analysis, the Weibull modulus $(m)$ and the characteristic strength $(\sigma c$, confidence interval of $95 \%$ ) were obtained through a diagram according to DIN ENV 843-5.19 The characteristic strength is the strength at a failure probability of approximately $63 \%$, and the Weibull modulus is used as a measure of the strength distribution, expressing the reliability of the material.

\section{Results}

\section{Phase transformation}

The autoclave aging led to the highest m-phase content values (AUT - 63.1\%, AUT+MechLoad $59.9 \%$ ), while mechanical cycling (MechLoad $3.8 \%$ ) promoted the lowest m-phase content (Table). It should be highlighted that even the storage under ambient conditions (room temperature $\pm 25^{\circ} \mathrm{C}$, without moisture, in a sealed plastic vessel) led to $\mathrm{m}$-phase content increase $(0 \%$ to $6.0 \%$, for 1 year), and the specimens submitted to storage in water during 1 year presented $17.61 \%$ of $\mathrm{m}$-phase content (Figure 1). 
Mechanical behavior of yttria-stabilized tetragonal zirconia polycrystal: Effects of different aging regimens

Table. Results (specific means, SD - standard deviations and $\mathrm{Cl}$ - confidence intervals) obtained from the different analysis.

\begin{tabular}{|c|c|c|c|c|c|}
\hline \multirow{2}{*}{ Groups } & \multirow{2}{*}{$\begin{array}{c}\text { XRD analysis } \\
\% \text { m-phase }\end{array}$} & \multicolumn{2}{|c|}{ Micro-scale roughness $(\mu \mathrm{m})$} & \multicolumn{2}{|c|}{ Weibull analysis } \\
\hline & & $\operatorname{Ra}(S D)$ & $\mathrm{Rz}(\mathrm{SD})$ & m $(95 \% \mathrm{Cl})$ & $\sigma_{\mathrm{c}}(\mathrm{MPa})(95 \% \mathrm{Cl})$ \\
\hline \multirow{2}{*}{ CTRL } & \multirow{2}{*}{0.0} & \multirow{2}{*}{$0.20(0.05)^{A}$} & \multirow{2}{*}{$1.70(0.40)^{\mathrm{A}}$} & 10.82 & 932.05 \\
\hline & & & & $(7.57-13.91)^{\mathrm{A}}$ & $(895.33-969.20)^{\mathrm{A}}$ \\
\hline \multirow{2}{*}{ AUT } & \multirow{2}{*}{63.1} & \multirow{2}{*}{$0.23(0.09)^{\mathrm{AB}}$} & \multirow{2}{*}{$1.82(0.56)^{A}$} & 21.43 & 1018.8 \\
\hline & & & & $(15.00-27.55)^{\mathrm{B}}$ & $(998.33-1039.11)^{\mathrm{B}}$ \\
\hline \multirow{2}{*}{ MechLoad } & \multirow{2}{*}{3.8} & \multirow{2}{*}{$0.21(0.03)^{A}$} & \multirow{2}{*}{$1.79(0.31)^{\mathrm{A}}$} & 10.01 & 953.9 \\
\hline & & & & $(7.00-12.86)^{\mathrm{A}}$ & $(913.36-995.13)^{\mathrm{AB}}$ \\
\hline \multirow{2}{*}{ AUT + MechLoad } & \multirow{2}{*}{59.9} & \multirow{2}{*}{$0.25(0.07)^{B}$} & \multirow{2}{*}{$1.97(0.47)^{\mathrm{A}}$} & 16.49 & 998.3 \\
\hline & & & & $(11.54-21.20)^{\mathrm{AB}}$ & $(972.26-1024.19)^{\mathrm{B}}$ \\
\hline \multirow{2}{*}{ STO } & \multirow{2}{*}{17.6} & \multirow{2}{*}{$0.23(0.08)^{A B}$} & \multirow{2}{*}{$1.90(0.51)^{\mathrm{A}}$} & 11.93 & 1078.85 \\
\hline & & & & $(8.35-15.33)^{A B}$ & $(1040.21-1117.80)^{c}$ \\
\hline
\end{tabular}

Same capital letters correspond to statistical similarity among same evaluated outcome (column); different capital letters correspond to statistical difference among same evaluated outcome (column).

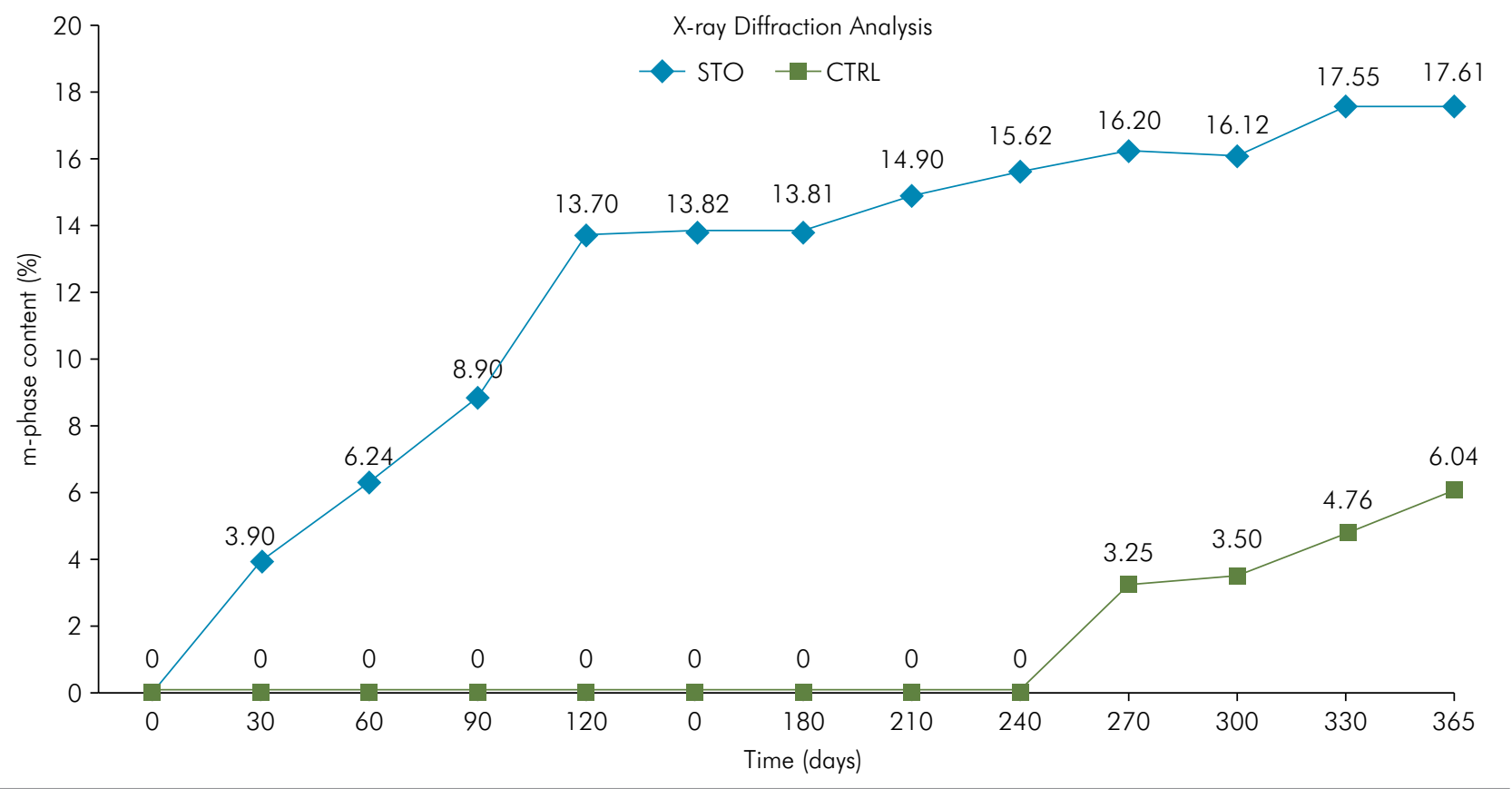

Figure 1. XRD data showing the $\mathrm{m}$-phase content increase in each month of storage in distilled water (STO group) in comparison to specimens kept on ambient conditions (sealed dry vessel at room temperature).

\section{Surface roughness}

There was no statistical difference for the $R z$ parameter among the evaluated conditions, while for $R a$, the AUT+MechLoad group presented statistically higher values (Table).

\section{Surface topography}

FE-SEM micrographics depict that all aging methods were unable to promote any considerable topographical alteration when compared to the as-sintered surface (Figure 2).

In relation to grain size, the as-sintered condition (CTRL) presented values ranging from 0.4 to $1.3 \mu \mathrm{m}$, similar to the group submitted to mechanical cycling (MechLoad - 0.5 to $1.3 \mu \mathrm{m}$ ). A larger range of grain sizes was observed in the groups that presented higher m-phase content (STO - 0.5 to $1.7 \mu \mathrm{m}$; AUT - 0.5 to $1.9 \mu \mathrm{m}$; AUT+MechLoad - 0.5 to $2.1 \mu \mathrm{m}$ ) (Figure 2). 

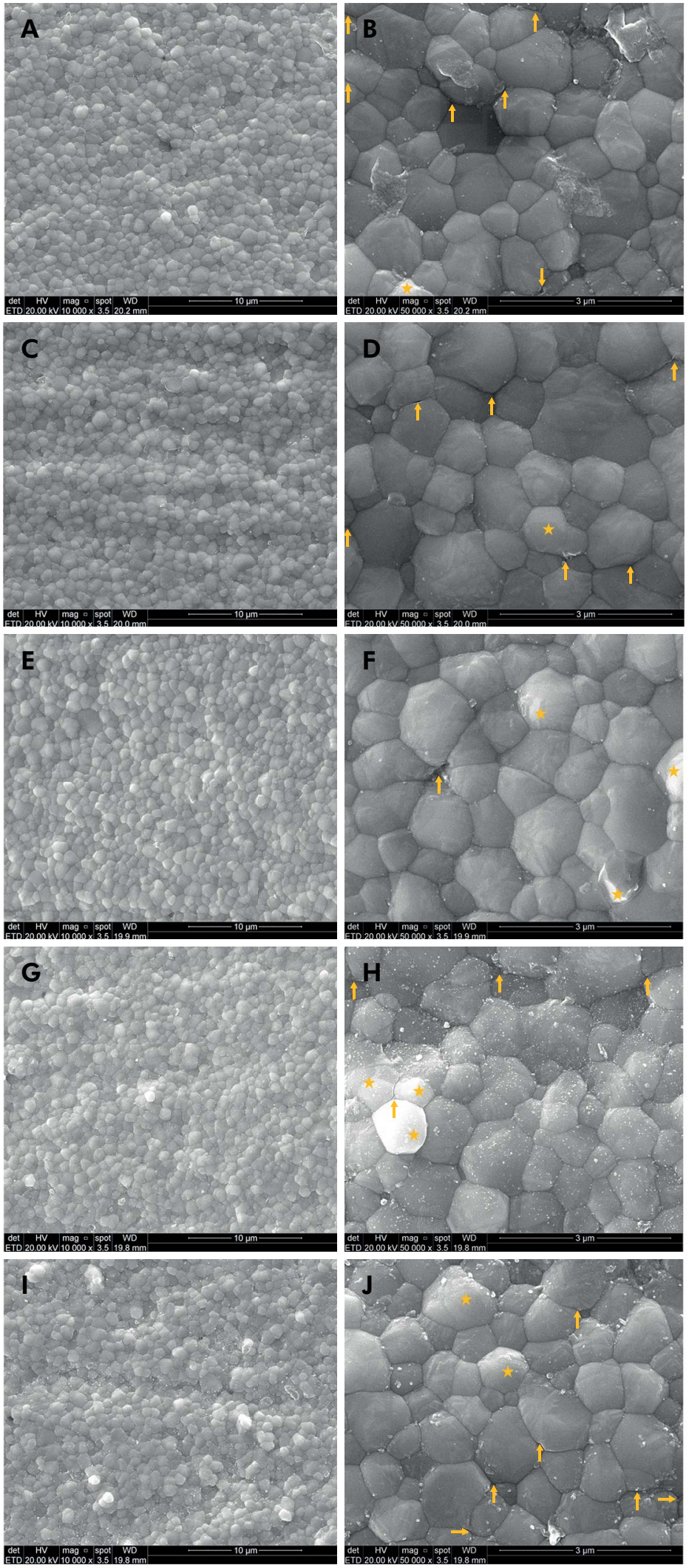

Figure 2. FE-SEM images (10,000x and 50,000x magnifications) of Y-TZP surface, demonstrating that no significant topographical alterations occurred after aging treatments. Yellow stars point to uplifted grains, and yellow arrows indicate intergranular microcracks. A and B - CTRL group, C and D - AUT, E and F - MechLoad, G and H - AUT+MechLoad, I and J - STO. 


\section{Biaxial flexural strength and reliability - Weibull Analysis}

Statistically higher values for strength were observed in the Storage group (1078.85 MPa), while the other conditions presented lower and similar values (CTRL: 932.05; MechLoad: 953.9; AUT+MechLoad: 998.3; AUT: $1018.8 \mathrm{MPa})$. For structural reliability (Weibull moduli), the AUT group ( $m=21.43$ ) presented increased values (Table; Figure 3); none of the other evaluated aging regimens promoted a deleterious effect compared with the control group.

\section{Qualitative fractographic analysis}

The fractographic patterns were similar for all groups, with the fracture origin located in a surface defect in the region subjected to tensile stresses during the flexural strength test, and propagating to the compression curl located on the subsurface of the piston load application area, at the compression side (Figure 4).

\section{Discussion}

Our findings support that the evaluated Y-TZP ceramic presents high resistance to aging effects, since none of the different aging regimens leading to different intensities of $\mathrm{t}-\mathrm{m}$ phase transformation promoted a deleterious effect on the surface roughness and mechanical properties of the material. Thus, the study hypothesis of a direct relation between aging, m-phase content increase and a deleterious impact on the evaluated outcomes was partially rejected. In fact, it might be highlighted that an increase in $\mathrm{m}$-phase content tended to promote also an increase in the material's structural reliability (Weibull moduli).

Topography analysis by FE-SEM showed no relevant alterations with aging. However, it is important to highlight the presence of intergranular cracks (Figure 2), even in the as-sintered condition. The presence of such cracks might occur due to two factors: 1, poor homogeneity during Y-TZP processing (i.e., variability in $\mathrm{Y}-\mathrm{TZP}$ powder homogeneity producing areas with higher or lower stabilizer content), resulting in unstable intergranular regions that, during sintering, ${ }^{20}$ result in the appearance of such cracks; 2 , in response to t-m phase transformation ${ }^{2}$ leading to the LTD effects.

Micro-defects and residual stresses can be introduced in the zirconia ceramic during its industrial production, lab preparations, and clinical adjustments. ${ }^{20}$ These micro-defects and residual stresses are cumulative,

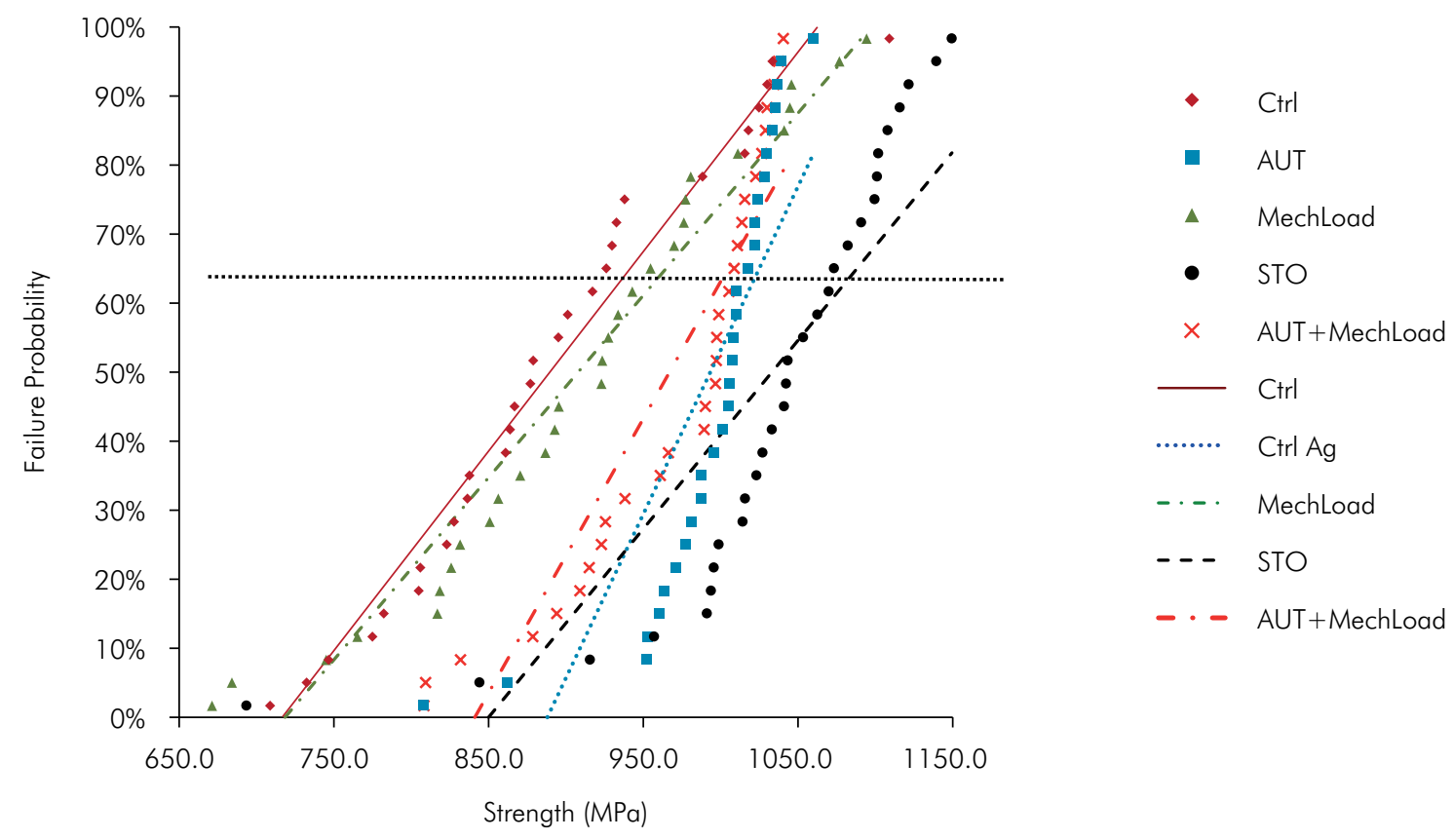

Figure 3. Weibull Plots from strength data (MPa) and failure probability. 

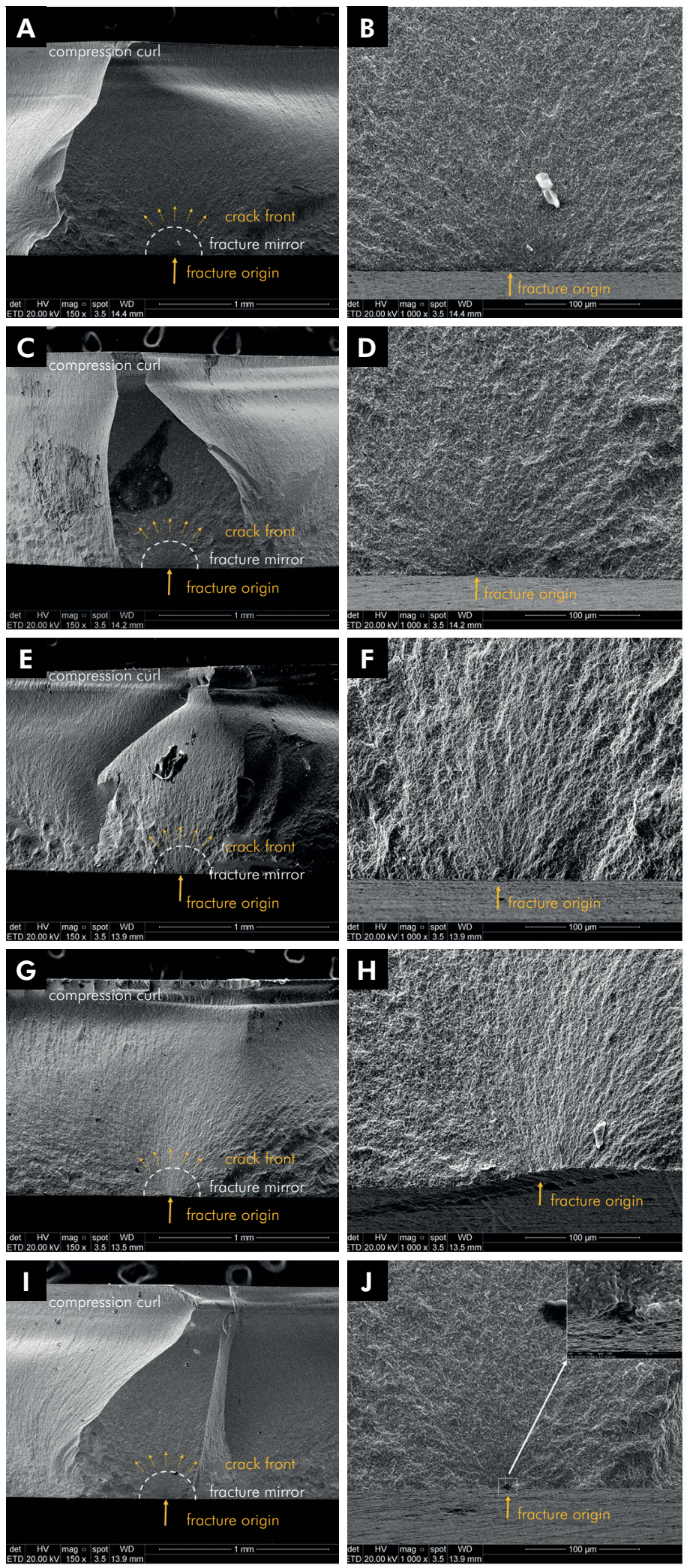

Figure 4. Fractographic analysis with FE-SEM $(150 \times$ and $1,000 \times$ magnifications), showing typical brittle fractured surfaces, with initial crack nucleation sites located on surface defects at the region of maximum tensile stress during the biaxial tests. In the right top of the 'J' image, the greater magnification $(10,000 \times)$ shows the defect on the crack nucleation site. A and B - CTRL group, C and D - AUT, E and F - MechLoad, G and H - AUT+MechLoad, I and J - STO. 
and might affect the final mechanical properties of the Y-TZP restorations.

The mechanism of LTD has been often described as an increase of internal stresses combined with water penetration inside the zirconia lattice, triggering a cascade of events. First, the transformation propagates inside of few grains and progressively enters into the surface and subsurface by a nucleation-and-growth (N-G) mechanism. Basically, the $t-m$ phase transformation of a few grains puts their neighbor grains under tensile stress, making them more susceptible to transform under the effect of water. ${ }^{2}$ Afterward, LTD proceeds into the material bulk and may jeopardize the strength, fracture toughness, and density of the Y-TZP. ${ }^{2,8}$ Thus, the aforementioned intergranular cracks (Figure 2) facilitate the water access inside the material (zirconia bulk) and may induce the $\mathrm{t}-\mathrm{m}$ phase transformation. Hence, the importance of the grain size factor is highlighted: larger tetragonal grain size leads to a lower stability to phase transformation. ${ }^{21,22}$

Despite not causing deleterious effects on the mechanical performance, the evaluated aging regimens promoted an extensive increase in $\mathrm{m}$-phase content and had a small influence on surface roughness (Table). The storage and the mechanical cycling regimens led to lower m-phase content, and resulted in a limited effect on roughness. Yet, it is important to emphasize that the m-phase transformation was triggered even when the samples were stored under ambient conditions ( $6.04 \%$ of $\mathrm{m}$-phase in 1 year), corroborating the effect of the long time storage on $\mathrm{m}$-phase transformation (Figure 1). To our knowledge, these events have been shown for the first time, and the outcomes may suggest that the increase in $\mathrm{m}$-phase content is unavoidable and accelerated by the presence of water.

Regarding autoclave aging, the increase in m-phase content was extensive, which probably led to more cracks on the materials' surface/subsurface. At the same time, this aging protocol was executed very rapidly and without mechanical stimuli. In response to that, it probably did not lead to grain pull-out, resulting in similar roughness values to the as-sintered condition. However, for the condition submitted to the additional intermittent mechanical loading
(AUT + MechLoad), the mechanical stimuli on the cracks produced during autoclave aging, led to an extensive grain lift and pullout, resulting in a rougher surface. This highlights the importance of combining autoclave and mechanical aging methods in Y-TZP ceramic studies, which is rarely considered in the existing literature.

Our data also support the concept of the toughening mechanism triggered by phase transformation, leading to residual stress concentration around superficial defects and arresting crack propagation, discussed by Hannink ${ }^{4}$ and extensively demonstrated in the literature. ${ }^{5,6,12,14,23,24}$ Pereira and collaborators ${ }^{8}$ stated that the critical point of $\mathrm{m}$-phase content for a deleterious effect on mechanical performance should be above $50 \%$. Our data show that the AUT+MechLoad group, even with $60 \% \mathrm{~m}$-phase content, had a statistically higher characteristic strength than the as-sintered condition.

However, it is also important to note that the highest characteristic strength was observed for the long-term storage condition (STO group) with approximately $18 \%$ of $\mathrm{m}$-phase content. Therefore, an inversion between toughening mechanism and low-temperature degradation effects might have occurred from $18 \%$ (STO) up to $60 \%$ in m-phase content (observed for AUT and AUT + MechLoad that also presented a higher characteristic strength than Ctrl group, but lower than STO group). Consequently, AUT and AUT + MechLoad conditions (combination of aging methods) could have probably led to a deleterious effect on mechanical properties.

The Weibull modulus describes the variation of strength values as the result of the flaw population in the material structure. Higher values of Weibull modulus indicate a more homogeneous flaw size distribution, less data scattering, and greater structural reliability. ${ }^{18}$ This study showed Weibull modulus ranging from 10 to 21.43 (Table; Figure 3), which is consistent with the values reported for ceramic crowns. ${ }^{14,25}$ We noticed a tendency for Weibull modulus increase after aging, since the groups with higher m-phase content tended to present higher Weibull modulus, which probably can be explained by the toughening mechanism that closes the crack tip, avoiding crack growth. In addition, a small difference in strength between specimens with 
large flaws and small flaws was observed, probably because of the rising of the toughness curve, which leads to higher toughness for larger flaws and lower toughness for smaller flaws.

Pinto et al. ${ }^{13}$ stated that, clinically, Y-TZP restorations are submitted to low biting forces (average of $80 \mathrm{~N}$ ), in a humid environment (simulated by autoclave cycles). They also consider that $10^{6}$ mechanical cycles correspond to 2 to 5 years of clinical service. Thus, from a mechanical performance viewpoint, the authors stated that the LTD would be a significant problem in a clinical scenario over a short/medium period of time (up to 5 years). However, new studies have proposed that the existing Y-TZP ceramics present high resistance to LTD and adequate clinical performance. ${ }^{14,24}$ Our data partially corroborates this last proposal, as the increase in $\mathrm{m}$-phase content, triggered by the aging regimens, did not impair the mechanical behavior of the zirconia material.

Besides, we have to consider that not all Y-TZP materials behave similarly when submitted to stimuli, as they present differences in terms of composition and grain size. The new generation of translucent zirconia has a higher concentration of yttria stabilizer that leads to a higher content of cubic phase, reducing or even avoiding the $\mathrm{t}-\mathrm{m}$ phase transformation mechanism and consequently increasing aging resistance (decreased LTD susceptibility). ${ }^{26}$

Despite the limitations of this in vitro study, our data clearly indicates that the evaluated Y-TZP ceramic presents high resistance to the effects of LTD. Based on our findings, future follow-up and time-dependent studies are recommended to evaluate critical flaw dimensions and fracture toughness data comparing different aging conditions; these additional data might also be used to determine the residual stress field on the specimen surfaces.

\section{Conclusions}

Within the limitations of this study and based on the findings, the following conclusions were drawn:

Increase in m-phase content seems to be unavoidable under the tested conditions;

The $\mathrm{t}-\mathrm{m}$ phase transformation proves to be a time-dependent spontaneous mechanism that is accelerated with the presence of stimuli (moisture, temperature, mechanical cycling), in different intensities;

None of the evaluated aging procedures impaired the mechanical behavior of the tested Y-TZP ceramic, which demonstrates the high resistance of the material to the effects of LTD;

An increase in m-phase content appears to induce an increase in the structural reliability (Weibull modulus) and characteristic strength of the material.

\section{Acknowledgements}

We thank CAPES (Agency for the High-Standard Promotion of Graduate Courses, Brazil) and CNPq agencies for supporting this study (scholarships).

\section{References}

1. Denry I, Kelly JR. Emerging ceramic-based materials for dentistry. J Dent Res. 2014;93(12):1235-42. https://doi.org/10.1177/0022034514553627

2. Cattani-Lorente M, Scherrer SS, Ammann P, Jobin M, Wiskott HW. Low temperature degradation of a Y-TZP dental ceramic. Acta Biomater 2011;7(2):858-65. https://doi.org/10.1016/j.actbio.2010.09.020

3. Chevalier J, Gremillard L, Deville S.

Low-Temperature degradation of zirconia and implications for biomedical implants.

Annu Rev Mater Res. 2007;37(1):1-32.

https://doi.org/10.1146/annurev.matsci.37.052506.084250
4. Hannink R, Kelly PM, Muddle BC. Transformation toughening in zirconia-containing ceramics. J Am Ceram Soc. 2000;83(3):461-87. https://doi.org/10.1111/j.1151-2916.2000.tb01221.x

5. Amaral M, Valandro LF, Bottino MA, Souza RO. Low-temperature degradation of a Y-TZP ceramic after surface treatments. J Biomed Mater Res B Appl Biomater 2013;101(8):1387-92. https://doi.org/10.1002/jbm.b.32957

6. Pereira GK, Muller C, Wandscher VF, Rippe MP, Kleverlaan CJ, Valandro LF. Comparison of different low-temperature aging protocols: its effects on the mechanical behavior of Y-TZP ceramics. J Mech Behav Biomed Mater.2016a;60:324-30. https://doi.org/10.1016/i.jmbbm.2016.02.017 
7. Kobayashi K, Kuwajima H, Masaki T. Phase change and mechanical properties of $\mathrm{ZrO} 2-\mathrm{Y} 2 \mathrm{O} 3$ solid electrolyte after ageing. Solid State lon. 1981;3-4:489-93. https://doi.org/10.1016/0167-2738(81)90138-7

8. Pereira GK, Venturini AB, Silvestri T, Dapieve KS, Montagner AF, Soares FZ et al. Low-temperature degradation of Y-TZP ceramics : a systematic review and meta-analysis. J Mech Behav Biomed Mater. 2015;55:151-63. https://doi.org/10.1016/i.jmbbm.2015.10.017

9. Inokoshi M, Vanmeensel K, Zhang F, De Munck J, Eliades G, Minakuchi $S$ et al. Aging resistance of surface-treated dental zirconia. Dent Mater. 2015;31(2):182-94. https://doi.org/10.1016/i.dental.2014.11.018

10. Lucas TJ, Lawson NC, Janowski GM, Burgess JO. Effect of grain size on the monoclinic transformation, hardness, roughness, and modulus of aged partially stabilized zirconia. Dent Mater. 2015;31(12):1487-92. https://doi.org/10.1016/i.dental.2015.09.014

11. Egilmez F, Ergun G, Cekic-Nagas I, Vallittu PK, Lassila LVJ. Factors affecting the mechanical behavior of Y-TZP. J Mech Behav Biomed Mater. 2014;37(15):78-87. https://doi.org/10.1016/i.jmbbm.2014.05.013

12. Cotes C, Arata A, Melo RM, Bottino MA, Machado JP, Souza RO. Effects of aging procedures on the topographic surface, structural stability, and mechanical strength of a $\mathrm{ZrO}$ based dental ceramic. Dent Mater 2014;30(12):e396-404. https://doi.org/10.1016/i.dental.2014.08.380

13. Pinto PA, Colas G, Filleter T, De Souza GM. Surface and mechanical characterization of dental yttria-stabilized tetragonal zirconia polycrystals (3Y-TZP) after different aging processes. Microsc Microanal. 2016;22(6):1179-88. https://doi.org/10.1017/S1431927616011843

14. Bergamo ETP, Silva WJ, Cesar PF, Del Bel Cury AA. Fracture load and phase transformation of monolithic zirconia crowns submitted to different aging protocols. Oper Dent. 2016;41(5):E118-30. https://doi.org/10.2341/15-154-L

15. International Organization for Standardization. ISO 6872-2015. Dentistry: ceramic materials. International Organization for Standardization. Geneve: International Organization for Standardization; 2015.

16. Guilardi LF, Pereira GK, Gündel A, Rippe MP, Valandro LF. Surface micro-morphology, phase transformation, and mechanical reliability of ground and aged monolithic zirconia ceramic. J Mech Behav Biomed Mater. 2017;65:849-56. https://doi.org/10.1016/i.jmbbm.2016.10.008

17. Toraya $\mathrm{H}$, Yoshimura MS. Calibration curve for quantitative analysis of the monoclinic tetragonal $\mathrm{ZrO} 2$ system by X-rays diffraction. J Am Ceram Soc. 1984;67(6):c1 19-21. https://doi.org/10.1111/i.1151-2916.1984.tb19715.x

18. Weibull W. A statistical distribution function of wide applicability. J Appl Mech. 1951;18:293-7.

19. Deutsch Institut für Normalization. DIN EN 843-5. Advanced technical ceramics: monolithic ceramics; mechanical tests at room temperature. Part 5: statistical analysis. Berlin: Deutsch Institut für Normalization; 2007.

20. Jing Z, Ke Z, Yihong L, Zhiijian S. Effect of multistep processing technique on the formation of micro-defects and residual stresses in zirconia dental restorations. J Prosthodont. 2014;23(3):206-12. https://doi.org/10.1111/jopr.12094

21. Lee TH, Lee SH, Her SB, Chang WG, Lim BS. Effects of surface treatments on the susceptibilities of low temperature degradation by autoclaving in zirconia. J Biomed Mater Res B Appl Biomater. 2012;100(5):1334-43. https://doi.org/10.1002/ibm.b.32700

22. Nakamura T, Usami $H$, Ohnishi $H$, Takeuchi $M$, Nishida $H$, Sekino $T$ et al. The effect of adding silica to zirconia to counteract zirconia's tendency to degrade at low temperatures. Dent Mater J. 2011;30(3):330-5. https://doi.org/10.4012/dmi.2010-142

23. Pereira GK, Fraga S, Montagner AF, Soares FZ, Kleverlaan CJ, Valandro LF. The effect of grinding on the mechanical behavior of Y-TZP ceramics: a systematic review and meta-analyses. J Mech Behav Biomed Mater. 2016b;63:417-42. https://doi.org/10.1016/j.jmbbm.2016.06.028

24. Monzavi M, Noumbissi S, Nowzari H. The Impact of in vitro accelerated aging, approximating 30 and 60 years in vivo, on commercially available zirconia dental implants. Clin Implant Dent Relat Res. 2017;19(2):245-52. https://doi.org/10.1111/cid.12462

25. Tinschert J, Zwez D, Marx R, Anusavice KJ. Structural reliability of alumina-, feldspar-, leucite-, mica- and zirconia-based ceramics. J Dent 2000;28(7):529-35. https://doi.org/10.1016/S0300-5712(00)00030-0

26. Stawarczyk B, Keul C, Eichberger M, Figge D, Edelhoff D, Lümkemann N. Three generations of zirconia: from veneered to monolithic. Part I. Quintessence Int. 2017;48(5):369-80. https://doi.org/10.3290/i.qi.a38057 